\title{
Current therapies for malignant pleural mesothelioma
}

\author{
Takashi Nakano
}

Received: 30 July 2007/ Accepted: 26 September 2007/Published online: 28 February 2008

(C) The Japanese Society for Hygiene 2008

\begin{abstract}
Mesothelioma is a highly lethal tumor derived from mesothelial cells, and its global incidence is increasing because of widespread exposure of numerous individuals to asbestos in the last 50 years. Mesothelioma is largely untreatable with any of the therapeutic modalities. Recently, a novel multitargeted antifolate pemetrexed has shown promising activity against malignant pleural mesothelioma, producing response rates of up to $40 \%$ when used in combination with cisplatin. In a large phase III study, use of a combination of pemetrexed and cisplatin was associated with significantly improved survival time and with greater antitumor activity compared with cisplatin alone. This combination also gave a significant response rate of approximately $50 \%$ in patients with epithelioid malignant pleural mesothelioma. These clinical benefits of pemetrexed-cisplatin doublet have changed the perception of mesothelioma chemotherapy. Other combinations, including gemcitabine in combination with cisplatin, have also shown encouraging response rates. Prognosis depends on gender, clinical stage of the tumor, histological subtype, platelet count, leukocyte counts, and performance status. Radiotherapy can palliate mesothelioma patients with chest pain, and has been indicated to be of benefit for the prevention of malignant seeding along the tract of a chest tube or needle biopsy. Trimodality treatment using extrapleural pneumonectomy, radiation and chemotherapy has shown
\end{abstract}

\section{T. Nakano}

Division of Respiratory Medicine, Department of Internal Medicine, Hyogo College of Medicine, 1-1 Mukogawa-cho, Nishinomiya, Hyogo 663-8501, Japan

T. Nakano $(\bowtie)$

Department of Thoracic Oncology, Hyogo College of Medicine, 1-1 Mukogawa-cho, Nishinomiya, Hyogo 663-8501, Japan e-mail: t-nakano@hyo-med.ac.jp promising therapeutic value. The development of chemotherapeutic regimens and the favorable outcomes of trimodality have led to new combined modality trials. In Japan, multicenter national trials against mesothelioma will begin in the near future.

Keywords Asbestos - Extrapleural pneumonectomy · Mesothelioma $\cdot$ Pemetrexed

\section{Introduction}

Malignant mesothelioma used to be a relatively uncommon disease, but its incidence has been rising in recent times and is predicted to peak around the year 2030 in Japan. There are currently 1,000 new mesothelioma cases per year. In June 2005, several inhabitants suffered from mesothelioma in Amagasaki, Hyogo, and their environmental exposure to asbestos resulted in medico-legal problems related to mesothelioma and social compensation for asbestos-related diseases in Japan. Since then, overwhelming media interest in the mesothelioma tragedy caused by asbestos has produced a high level of awareness of the risks of asbestos and mesothelioma.

Mesothelioma occurs at different anatomical sites that contain mesothelial cells. The most common site is pleura, followed by the peritoneum, the pericardium, and the male genitalia. Malignant pleural mesothelioma (MPM) is a highly lethal and particularly refractory tumor for which conventional chemotherapeutic regimens have been far from satisfactory in achieving a clinical response. However, the role of chemotherapy in MPM has gradually changed since the late 1990s, with the emergence of new active agents and evidence that chemotherapy palliates clinical symptoms. The majority of patients have clinical 
Table 1 The International Mesothelioma Interest Group (IMIG) staging system

T1 T1a Tumor limited to the ipsilateral parietal pleura, including mediastinal and diaphragmatic pleura No involvement of the visceral pleura

T1b Tumor involving the ipsilateral parietal pleura, including mediastinal and diaphragmatic pleura Scattered foci of tumor also involving the visceral pleura

T2 Tumor involving each of the ipsilateral pleura surfaces (parietal, mediastinal, diaphragmatic, and visceral pleura) with at least one of the following features:

Involvement of diaphragmatic muscle

Confluent visceral pleural tumor (including the fissures) or extension of tumor from visceral pleura into the underlying pulmonary parenchyma

T3 Describes locally advanced but potentially resectable tumor

Tumor involving all of the ipsilateral pleural surfaces (parietal, mediastinal, diaphragmatic, and visceral pleura) with at least one of the following features:

Involvement of the endotharacic fascia

Extension into the mediastinal fat

Solitary, completely resectable focus of tumor extending into the soft tissues of the chest wall

Nontransmural involvement of the pericardium

T4 Describes locally advanced technically unresectable tumor

Tumor involving all of the ipsilateral pleural surfaces (parietal, mediastinal, diaphragmatic, and visceral pleura) with at least one of the following features:

Diffuse extension or multifocal masses of tumor in the chest wall, with or without associated rib destruction

Direct transdiaphragmatic extension of tumor to the peritoneum

Direct extension of tumor to the contralateral pleura

Direct extension of tumor to one or more mediastinal organs

Direct extension of tumor into the spine

Tumor extending through to the internal surface of pericardium, with or without a pericardial effusion; or tumor involving the myocardium

N-lymph

nodes

Nx Regional lymph nodes cannot be assessed

N0 No regional lymph node metastases

N1 Metastases in the ipsilateral bronchopulmonary or hilar lymph nodes

N2 Metastases in the subcarinal or the ipsilateral mediastinal lymph nodes, including the ipsilateral internal mammary nodes

N3 Metastases in the contralateral mediastinal, contralateral internal mammary, ipsilateral, or contralateral supraclavicular lymph nodes

M- metastases

Mx Presence of distant metastasis cannot be assessed

M0 No distant metastasis

M1 Distant metastasis present

\begin{tabular}{ll}
\hline Stage & Description \\
\hline Ia & $\mathrm{T}_{1 \mathrm{a}} \mathrm{N}_{0} \mathrm{M}_{0}$ \\
Ib & $\mathrm{T}_{1 \mathrm{~b}} \mathrm{~N}_{0} \mathrm{M}_{0}$ \\
II & $\mathrm{T}_{2} \mathrm{~N}_{0} \mathrm{M}_{0}$ \\
III & AnyT $_{3} \mathrm{M}_{0}$ \\
& AnyN $_{1} \mathrm{M}_{0}$ \\
& $\mathrm{AnyN}_{2} \mathrm{M}_{0}$ \\
IV & AnyT $_{4}$ \\
& AnyN $_{3}$ \\
& AnyM \\
\end{tabular}

stage Ib or more locally advanced disease, as defined by the International Mesothelioma Interest Group (IMIG; Table 1; [1]). These patients do not benefit from radical surgery; therefore, most patients are candidates for chemotherapy at some point in their treatment. Patients receiving best supportive care alone have short survivals 
and suffer from progressive pain. Recent results from the first large randomized, international phase III trial of a multitargeted antifolate, pemetrexed combined with cisplatin, demonstrated that the combination significantly improved response rates, time to progression, and overall survival in comparison with cisplatin alone [2]. Also, a more recent trial demonstrated that cisplatin in combination with raltitrexed significantly improved median survival compared to single-agent cisplatin (11.4 vs. 8.8 months) [3]. MPM is a locally aggressive tumor within and around the thoracic cavity and it uncommonly exhibits clinical features of metastatic malignancy. The singletreatment modality has not been effective; however, multimodality approaches have demonstrated an improvement in survival and quality of life [4].

\section{Asbestos consumption and mesothelioma in Japan}

Development of malignant mesothelioma is strongly associated with asbestos exposure, with $80 \%$ of patients previously exposed to asbestos fiber. In Japan, the incidence of malignant mesothelioma is continuing to rise, with a peak in the epidemic expected around the year 2030. A very small amount of asbestos, mainly chrysotile, was produced in Japan; therefore, the amount of asbestos imported is nearly equal to the total used. In the history of the Japanese asbestos industry, 30,000-40,000 tons was imported per year before World War II. Thereafter, the import of asbestos ceased between 1942 and 1948 because of the economic blockade of Japan during World War II and the complete destruction of its industry as a result of the war. The recovery of Japanese industry has been accelerating since the Korean War (1950-1953), and the import of asbestos vastly increased in the late 1960s. In contrast to the situation in Japan, the use of asbestos in the United States increased rapidly during and soon after World War II, at peaking from the 1950s through to 1970 . The US imported 165,000 tons of chrysotile in 1935 and 650,000 tons in 1962. Starting in 1970, the consumption of asbestos declined precipitously in the US as a result of its carcinogenicity, thus accounting for the decreasing incidence rate of mesothelioma in the twenty-first century there. The number of asbestos lawsuits increased dramatically during the early 1970s. In contrast, more than 300,000 tons of asbestos were continuously imported between 1973 and 1977 in Japan. After asbestos spraying was prohibited in 1974, asbestos consumption began to decrease in Japan. However, from the mid-1980s, one economic policy of the Japanese government was to decrease Japan's trade surplus, markedly increasing demand for building construction. The import of asbestos therefore rose again to 320,000 tons in 1988, but dropped again to 176,000 tons in 1997 , because of the serious recession in the Japanese economy. Therefore, compared to the US, there is a time-lag of about 20 years in the decrease in use of asbestos. The last decades of the twentieth century saw stabilization and/or declines in mesothelioma rates in the US. However in Japan, the annual numbers of deaths from mesothelioma increased markedly from 500 cases in 1995 to 953 cases in 2004; this increase occurred in parallel with the continued importation of asbestos with a delay of 40 years, due to the long latency period of mesothelioma (Fig. 1). Considering the long latency period of about 40 years and the more than 300,000 tons of asbestos used between 1973 and 1977 in Japan, the incidence of malignant mesothelioma can be expected to increase dramatically in the near future, possibly from 2010 onwards.
Fig. 1 Asbestos imports and annual number of deaths from mesothelioma in Japan

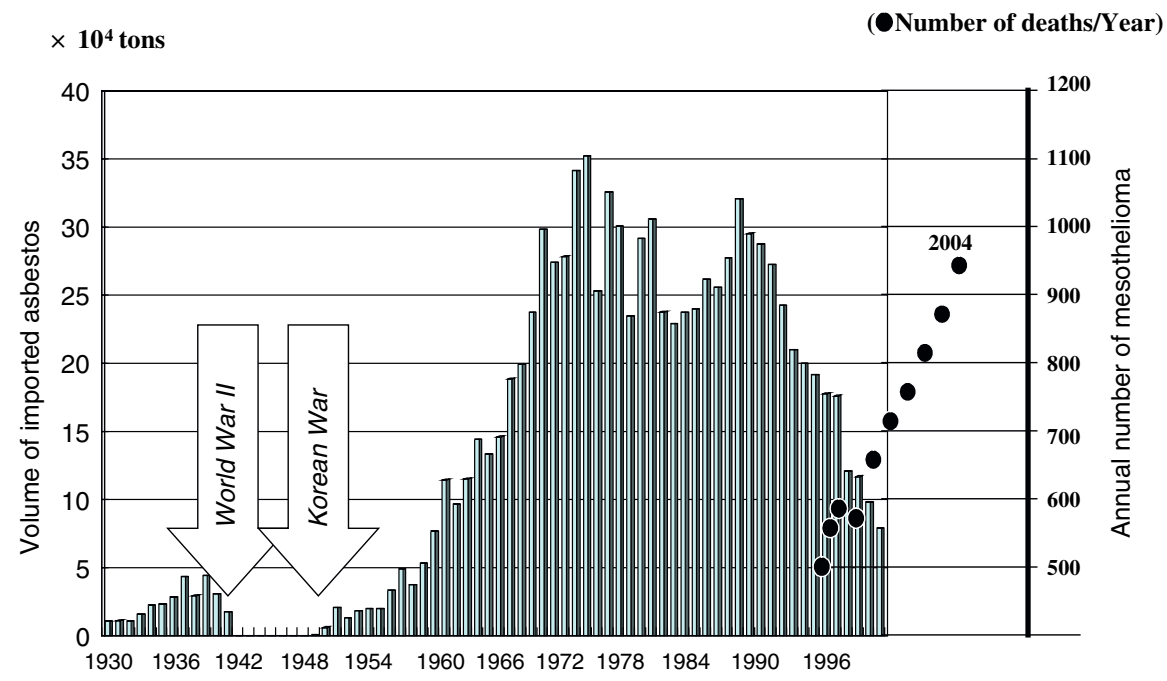




\section{First-line chemotherapy for malignant pleural mesothelioma}

MPM is a highly lethal tumor and is poorly responsive to any conventional chemotherapy. Over the last few decades, numerous clinical studies have been performed to identify single agents or combinations of them that possess any activity against MPM; however, no standard regimen has emerged. Many of the phase II studies have been small, single-institution trials because of the rarity of the neoplasm, and the patients enrolled are usually heterogeneous in prognostic factors, which may lead to statistical bias and misleading interpretations. In the past, several small phase II trials have reported relatively positive results. However, these favorable outcomes have usually not been confirmed by larger confirmatory trials.

Cytotoxic agents, including anthracyclines, platinum derivatives, topoisomerase I inhibitors and antimetabolites, have demonstrated some activity against MPM [5, 6], although their single-agent response rates are disappointingly low. Several combinations of these agents have been tested in many phase II studies, but the role of drug combinations in the treatment of MPM remains unclear. A response rate of $13-14 \%$ can be achieved by using cisplatin as a single agent against MPM. In a meta-analysis of studies published between 1965 and 2001, cisplatin was found to be the most active agent [7]. Treatment regimens in which one further active agent is added to cisplatin have been used in attempts to enhance anti-mesothelioma activity. Carboplatin was not superior to cisplatin; single-agent response rates were similar to those obtained with conventional doses of cisplatin (6$16 \%$ ) [8]. A platinum analog, oxaliplatin, has only been tested in combination with raltitrexed (response rate $=$ $35 \%$ ) [9], or with gemcitabine (GEM) (response rate $=$ $40 \%$, median survival $=13$ months) [10]; it has not been tested as a single agent. However, when combined with vinorelbin, oxaliplatin significantly increased in toxicity and did not appear to offer any advantage over vinorelbine alone [11]. The most widely tested combination is the cisplatin-doxorubicin (DXR) doublet, which has given response rates of $15-25 \%$ [12]. DXR was once believed to be one of the most active agents against MPM; however, the response rate to single-agent DXR was reported to be $14 \%$, with a median survival of 7.3 months. The Cancer and Leukemia Group B (CALGB) tested combinations of cisplatin plus DXR and cisplatin plus mitomycin C (MMC) in a randomized trial [13]. A $14 \%$ response rate was achieved using cisplatin plus DXR, and a $26 \%$ response rate was achieved using cisplatin plus MMC. The triplet of cisplatin-DXR-MMC had a response rate of $20.9 \%$ against mesothelioma. The activity of this triplet was similar to those of the respective doublets [14]. Berghmans et al. [7] reviewed 83 studies with 88 treatment arms for MPM from 1965 to 2001, and noted that cisplatin was the most active single agent and that the cisplatin-DXR doublet had the highest response rate, of $28.5 \%$. This combination was recommended as the control arm for subsequent trials.

Irinotecan (CPT-11) is a potent topoisomerase I inhibitor with activity against various tumors, which has been evaluated in MPM. There were no responses with singleagent CPT-11 in a phase II trial of the CALGB [15]. CPT11 is converted to its more active metabolite, SN-38, which has 1,000 times the potency of the parent compound. Intravenous administration of CPT-11 at a dose of $60 \mathrm{mg} /$ $\mathrm{m}^{2}$ can produce distributions of CPT-11 and SN-38 into the pleural fluid that allow the more active $\mathrm{SN}-38$ to come into contact with mesothelioma cells in the thoracic cavity for a longer time and at a higher concentration than for CPT-11 [16]. It is also expected that CPT-11 is more active in combination with other agents against MPM. The CPT-11cisplatin doublet produced response rates of $26.7 \%$ in MPM [16] and 24\% in malignant peritoneal mesothelioma [17], and the CPT-11-cisplatin-MMC triplet produced a response rate of $35 \%$ and progression-free survival of 6.5 months [18].

Antimetabolites, such as the antifolates (methotrexate, edatrexate and trimetrexate), are among the most active agents for the treatment of MPM. Halme et al. have demonstrated that high-dose methotrexate in combination with interferon $\alpha$ or $\gamma$ had a response rate of $29 \%$ and a median survival of 17 months [19]. Other antifolates, including trimetrexate and edatrexate, did not show a clear benefit.

Although GEM, a pyrimidine antimetabolite, achieves limited activity as a single agent (response rates of $0-7 \%$ ), its combination with cisplatin shows an increased response. Byrne et al. [20] reported a promising response rate of $47.6 \%$ with the combination of cisplatin and GEM in a four-week schedule. They performed a multicenter trial of the same regimen, and confirmed its anti-mesothelioma activity (response rate $=33 \%$ ) [21]; however, a subsequent trial of similar design but using a slightly higher planned dose intensity of GEM failed to duplicate this result, demonstrating a response rate of $16 \%$ [22].

Pemetrexed has demonstrated a single-agent response rate of $14 \%$ with acceptable toxicity. Two phase III randomized trials have been reported with promising results. The international trial of pemetrexed with cisplatin versus cisplatin alone in chemotherapy-naïve MPM patients demonstrated a significant increase in overall survival (12.1 vs. 9.3 months), a longer median time to progression (5.7 vs. 3.9 months), and a greater response rate (41 vs. 17\%) for the combination of pemetrexed and cisplatin with supplementation of vitamin $\mathrm{B}_{12}$ and folic acid [2]. Adverse 
effects of pemetrexed can be minimized with simultaneous supplementation of folic acid and vitamin $\mathrm{B}_{12}$. This supplementation does not diminish the efficacy and may improve clinical outcome [23]. Pulmonary function tests and quality of life were also better in the pemetrexedcisplatin arm. Also, the combination of raltitrexed and cisplatin was reported to improve overall survival compared with cisplatin in a first-line treatment of 250 patients [3], confirming that cisplatin with an antifolate should be the reference regimen in patients with MPM. In the firstline setting, it has generally been accepted that the combination of pemetrexed and cisplatin should be considered standard for MPM.

\section{Second-line chemotherapy for mesothelioma}

Clinical trials of anticancer agents against MPM have almost exclusively focused on chemotherapy-naïve patients. Those who have had clinical benefit from firstline chemotherapy are often still in good performance status when radiological progression is documented, and commonly have expectations for second-line treatment. However, there is no current standard second-line chemotherapeutic regimen for MPM following the breakthrough outcome of the treatment with the pemetrexed-cisplatin doublet [2]. Manegold et al. [24] examined the use of poststudy chemotherapy of the patients treated in the previously described phase III trial. Second-line chemotherapy was administered to $37 \%$ of the patients treated with the pemetrexed plus cisplatin arm and $47 \%$ of the patients treated with the cisplatin arm. The most commonly used second-line agents have been GEM, or the combination of GEM and cisplatin. After adjusting for prognostic factors and the first-line treatment group, a multiple regression analysis indicated that post-study chemotherapy was significantly associated with prolonged survival. The striking key finding of this report was the survival advantage observed in the pemetrexed plus cisplatin treatment group in spite of the imbalance in post-study chemotherapy. In second-line treatment of non-small cell lung cancer, pemetrexed was approved partly because it had a favorable toxicity pattern compared with docetaxel. Some secondline studies have been performed for mesothelioma. Porta et al. [25] reported that the antimetabolite raltitrexed in combination with oxaliplatin was inactive as a second-line treatment for MPM. There is currently insufficient evidence to recommend second-line chemotherapy for patients with a good performance status who progress following first-line treatment. Patients with adequate performance status should be enrolled into a clinical trial of second-line treatment.
Combination chemotherapy represents the standard treatment for the patients with local advanced mesothelioma who are not amenable to any local approach, such as extrapleural pneumonectomy (EPP). Novel and more effective therapeutic strategies are needed for mesothelioma. At present, the regimen of the combination of pemetrexed and cisplatin is the first-line standard medical treatment for MPM.

\section{Radiological evaluation of tumor response}

The impact of chemotherapy in mesothelioma has been difficult to evaluate because of the rarity of the tumor, the relatively small number of randomized studies, the heterogeneity of the pathologic subtypes, and the difficulties in assessing tumor response using CT scan. Radiological measurement of the tumor is sometimes difficult in MPM
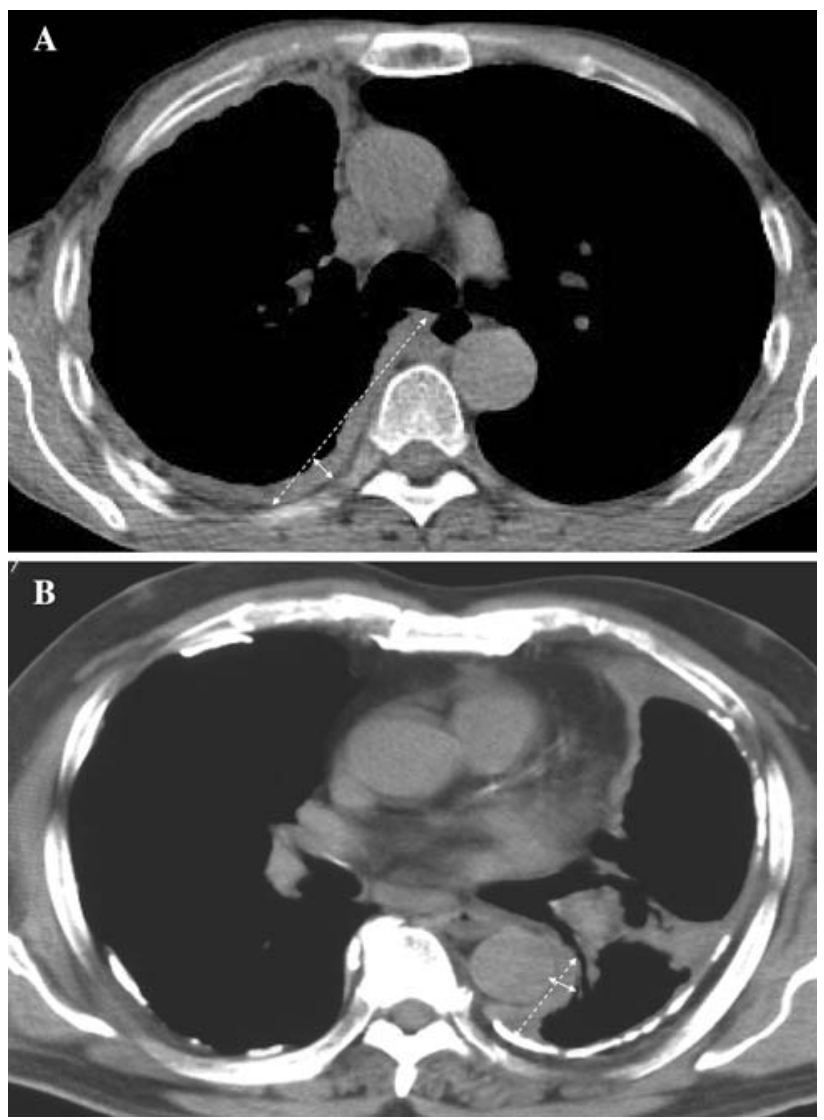

Fig. 2A-B RECIST and modified RECIST criteria. Malignant mesothelioma of the right thoracic cavity (A); diffuse pleural thickening estimated by RECIST (dotted line), and modified RECIST (solid line). Mesothelioma of the left thoracic cavity (B); diffuse pleural thickening and multiple calcified pleural plaques, thickness estimated by RECIST (dotted line), and modified RECIST (solid line) 
because of the diffuse spread of the tumor along the inner thoracic wall and the variable volumes of pleural effusion that obscure its actual extent (Fig. 2A,B). The guidelines of measurement offered by WHO or RECIST (Response Evaluation Criteria in Solid Tumors) are not appropriate for mesothelioma, given its circumferential growth pattern [26, 27]. Accordingly, modified RECIST criteria have been proposed specifically for MPM, in which between one and three unidimensional measurements of tumor thickness perpendicular to vital structures, such as the chest wall, the vertebral column and mediastinum, are obtained on each of three separate CT sections [2, 28]. It may be simpler and more reproducible than the RECIST criteria. The sum of these unidimensional measurements is used to represent tumor burden, and assessed according to the RECIST response classification criteria. Nodal, subcutaneous, and other bidimensionally measurable lesions are measured unidimensionally as per the RECIST criteria. Recently Armato et al. [29] have developed a computer-assisted technique for mesothelioma tumor thickness measurements, and have shown that their technique makes the radiological assessment of mesothelioma more efficient and consistent.

Recently, combined $\left({ }^{18} \mathrm{~F}\right)$ fluorodeoxyglucose positron emission tomography (FDG-PET) and CT have been used in the diagnosis of MPM (Fig. 3). In the past few years, FDG-PET has been used to monitor response to treatment in several neoplasms. Low standardized uptake value (SUV) and epithelioid histology indicate the best survival, whereas high SUV and nonepithelioid histology indicate the worst survival. Ceresoli et al. [30] demonstrated that in MPM treated with pemetrexed or the pemetrexed-cisplatin doublet an early reduction of FDG uptake, as measured by SUV max relative change, was significantly correlated to patient outcome. However, this approach has not yet been fully validated.

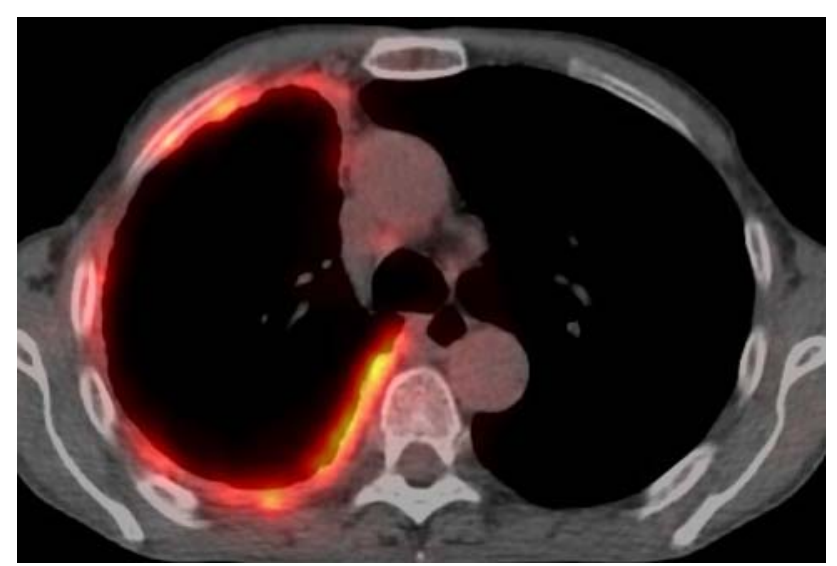

Fig. 3 Integrated CT-PET in patient with malignant pleural mesothelioma showing diffuse uptake of FDG in right pleural tumor

\section{Radiotherapy for malignant pleural mesothelioma}

Mesothelioma cells are unexpectedly sensitive to radiation compared to non-small cell lung cancer cells in vitro [31]; however, radiotherapy for patients with MPM is limited because of the large target volume and the radiosensitivity of the adjacent lung, heart, mediastinum, liver, and spinal cord. The role of radiotherapy in MPM is defined by symptomatic relief and palliation, prevention of neoplastic cell seeding, and adjuvant therapy following EPP. Radiotherapy is most commonly used to palliate pain in advanced MPM, but the effect of radiation monotherapy on prolonging survival is minimal. Pain is fairly well controlled with radiotherapy doses above $40 \mathrm{~Gy}$. Chest wall seeding following the previous invasive procedures, such as pleural aspiration, biopsy, thoracoscopy, and thoracostomy is a characteristic clinical feature of MPM. Prophylactic radiotherapy at the dose of 21 Gy in three fractions has been indicated to be of benefit for the prevention of malignant seeding along the tracts $[32,33]$. However, a randomized study showed no benefit of a dose of $10 \mathrm{~Gy}$ in a single fraction in preventing procedure tract metastasis [34]. Radiotherapy has often been added to surgery in an attempt to improve local control. It is used as adjuvant therapy after EPP as part of trimodality treatment, which is effective at reducing local recurrence of the tumor and improving overall survival [35].

Radiotherapy with an attempt to treat the entire involved pleural surface at a potentially curative dose $(>60 \mathrm{~Gy})$ is technically very difficult because of a high risk of radiation pneumonitis, myelitis, hepatitis and myocarditis. EPP removes radiation-sensitive lung, so it permits the delivery of higher postoperative doses to the involved hemithorax. A phase II trial conducted at the Memorial Sloan-Kettering Cancer Center demonstrated that a high dose of hemithoracic radiation (54 Gy) following EPP was well tolerated with reduced local recurrence rates and prolonged survival for early-stage MPM [35]. A new solution that produces more conformal dose distributions by saving critical structures is provided by intensity-modulated radiotherapy (IMRT) [36]. Forster et al. [37] reported using IMRT after EPP in seven MPM and saw no local recurrence during 13 months of follow-up. The combined use of postoperative IMRT and EPP has been suggested to improve overall survival and reduce recurrence. Recently, it has been reported that standard-dose IMRT after EPP and adjuvant chemotherapy resulted in a high rate $(46 \%)$ of fatal pneumonitis [38]. Thereafter, a new system for calculating thoracic IMRT doses was established in the MD Anderson Cancer Center [39].

The role of radiotherapy in the treatment of MPM has yet to be fully defined. Many of the previous studies on radiotherapy have been small and retrospective. 
Prospective randomized trials are needed to evaluate definitive radiotherapy in combination with novel radiosensitizers and chemotherapy in MPM.

\section{Surgical and multimodality approaches to the management of malignant pleural mesothelioma}

Two surgical interventions are used in the management of MPM: EPP and pleurectomy-decortication (P/D). EPP is aggressive surgical procedure which removes, en bloc, the entire parietal pleura, visceral pleura, lung, pericardium, and hemidiaphragm. $\mathrm{P} / \mathrm{D}$ is a debulking procedure that removes the involved parietal and visceral pleura and possibly the diaphragm, providing an alternative cytoreductive option for patients unfit for EPP. The lung is not removed, so complete tumor resection cannot be achieved if the interlobar fissure is involved. EPP offers a potential complete tumor resection, and adjuvant radiotherapy can be given at high dose. The number of operable MPM patients is still rather small; $20-30 \%$ of patients diagnosed with MPM are candidates for P/D or EPP at the time of diagnosis [40]. The role of surgery for MPM with the intension of cure continues to be a matter of debate [41]; however, EPP is the most effective therapy for achieving local control. Prolongation of survival has been reported in MPM patients undergoing EPP followed by adjuvant therapies. The Brigham and Women's Hospital/Dana Farber Cancer Institute Group in Boston has reported promising results from a trimodality combining EPP with sequential adjuvant chemotherapy using a regimen of DXR + cyclophosphamide + cisplatin initially, and paclitaxel + carboplatin in later patients, as well as radiotherapy (55 Gy), and they report that the median survival in the 176 patients was 19 months and the two- and five-year survival rates were 38 and $14 \%$, respectively [42]. The most recent follow-up data from this group reported an operative mortality rate for EPP of $4 \%$, with a major morbidity rate of 24\% [42]. In contrast, McCormack et al. [43] reported an operative mortality of $1.8 \%$ for P/D. The response rate of the cisplatin-GEM doublet has been reported to range between 16 and $47 \%$, and was $32 \%$ in a neo-adjuvant trial [44]. The response to the pemetrexed-cisplatin doublet has been reported to be $41 \%$ in advanced MPM, so most groups are now exploring this combination in a neo-adjuvant setting.

Before a MPM patient is considered for EPP, judgment is required to determine whether the patient can tolerate radical surgical resection and induction chemotherapy. Evaluation of clinical prognostic factors is also required; a worse outcome has been associated with the male gender, $>75$ years of age, poor PS, platelet count $>400,000 / \mathrm{mm}^{3}$, serum LDH $>500 \mathrm{IU} / \mathrm{L}$, low hemoglobin level and weight loss. The criteria for selecting patients for EPP remains a matter of debate. For patients being considered for EPP, a predicted postoperative FEV1.0 of less than $1 \mathrm{~L}$ is a contraindication to EPP. MPM patients with unfavorable characteristics such as N2 disease (Table 1) and sarcomatoid histology would not be candidates for enrollment in a trimodality clinical trial. Most trimodality trials for MPM have included patients with IMIG T3 disease (Table 1), if the extent of chest wall invasion was such that the thoracic surgeon deemed resection to be possible. Both EPP and $\mathrm{P} / \mathrm{D}$ are technically complex and are not frequently performed by most surgeons. EPP is associated with significant rates of morbidity and mortality, so MPM patients may benefit from referrals to institutions with significant experience with the procedure.

\section{Conclusion}

Chemotherapy for MPM is now starting to have a clinical benefit. The recent phase III trial demonstrated significant improvements in survival and disease-related symptoms for those patients treated with the pemetrexed-cisplatin doublet. In January 2007, pemetrexed-cisplatin was approved for the treatment of MPM by the Ministry of Health, Labour and Welfare in Japan. Now we have a standard chemotherapeutic regimen against MPM. Several multicenter national trials against mesothelioma will also begin in the near future in Japan.

\section{References}

1. International Mesothelioma Interest Group. A proposed new international TNM staging system for malignant pleural mesothelioma. Chest. 1995;108:1122-8.

2. Vogelzang NJ, Rusthoven JJ, Symanowski J, Symanowski J, Denham C, Kaukel E, et al. Phase III study of pemetrexed in combination with cisplatin versus cisplatin alone in patients with malignant pleural mesothelioma. J Clin Oncol. 2003;21:2636-44.

3. Van Meerbeeck JP, Gaafar R, Manegold C, van Klaveren RV, Van Marck EA, Vincent M, et al. Randomized phase III study of cisplatin with or without raltitrexed in patients with malignant pleural mesothelioma: an intergroup study of the European Organization for Research and Treatment of Cancer Lung-Cancer Group and the National Cancer Institute of Canada. J Clin Oncol. 2005;23:6881-9.

4. Martino D, Pass HI. Integration of multimodality approaches in the management of malignant pleural mesothelioma. Clin Lung Cancer. 2004;5:290-8.

5. Tomek S, Manegold C. Chemotherapy for malignant pleural mesothelioma: past results and recent developments. Lung Cancer. 2004;455:S103-19.

6. Steele JPC Rudd RM. Systemic chemotherapy for malignant pleural mesothelioma. In: O'Byrne K, Rusch V, editors. Malignant Pleural Mesothelioma. New York: Oxford; 2006. p. 298313. 
7. Berghmans T,Paesmans M, Lalami Y, Louviaux I, Luce S, Mascaux C, et al. Activity of chemotherapy and immunotherapy on malignant mesothelioma: a systemic review of the literature with meta-analysis. Lung Cancer. 2002;38:111-21.

8. Tomek S, Emri S, Krejcy K, Manegold C. Chemotherapy for malignant pleural mesothelioma: past results and recent developments. Br J Cancer. 2003;88:167-74.

9. Fizazi K, Caliandro R, Soulie P, Fandi A, Daniel C, Bedin A, et al. Combination raltitrexed (Tomudex ${ }^{\circledR}$-oxaliplatin: a step forward in the struggle against mesothelioma? The Institut Gustave Roussy experience with chemotherapy and chemoimmunotherapy in mesothelioma. Eur J Cancer. 2000;36:1514-21.

10. Schuette W, Blankenburg T, Lauerwald K, Schreiber J, Bork I, Wollscgkaeger B, et al. A multicenter phase II study of gemcitabine and oxaliplatin for malignant pleural mesothelioma. Clin Lung Cancer. 2003;4:294-7.

11. Fennell DA, Steele C, Shamush J, Sheaff MT, Evans MT, Goonewardene TI, et al. Phase II trial of vinorelbine and oxaliplatin as first-line therapy in malignant pleural mesothelioma. Lung Cancer. 2005;47:277-81.

12. Ong ST, Vogelzang NJ. Chemotherapy in malignant pleural mesothelioma: a review. J Clin Oncol. 1996;14:1007-17.

13. Chahinian AP, Antman K, Goutsou M, Corson JM, Suzuki Y, Modeas C, et al. Randomized phase II trial of cisplatin with mitomycin or doxorubicin for malignant mesothelioma by the Cancer and Leukemia Group B. J Clin Oncol. 1993;11:1559-65.

14. Pennucci MC, Ardizzoni A, Pronzato P, Fioretti M, Lanfranco C, Verna A, et al. Combined cisplatin, doxorubicin, and mitomycin for the treatment of advanced pleural mesothelioma: a phase II FONICAP trial. Italian Lung Cancer Task Force. Cancer. 1997;79:1897-902.

15. Kindler HI, Herndon JE, Zhang C, Green MR. Cancer and Leukemia Group B. Irinotecan for malignant mesothelioma. A phase II trial by the Cancer and Leukemia Group B. Lung Cancer. 2005;48:423-8

16. Nakano T, Chahinian AP, Shinjo M, Togawa N, Tonomura A, Miyake M, et al. Cisplatin in combination with irinotecan in the treatment of patients with malignant pleural mesothelioma. A pilot phase II clinical trial and pharmacokinetic profile. Cancer. 1999;85:2375-84.

17. Le DT, Deavers M, Hunt K, Malpica A, Verschraegen CF. Cisplatin and irinotecan (CPT-11) for peritoneal mesothelioma. Cancer Invest. 2003;21:682-9.

18. Fennell DA, Steele JP, Shamash J. A phase II study of irinotecan, cisplatin, and mitomycin $\mathrm{C}$ in malignant pleural mesothelioma. Lung Cancer. 2003;41(Suppl 2):S221b.

19. Halme M, Knuuttila A, Vehmas T, Tammilehto L, Mantyla M, Salo J, et al. High-dose methotrexate in combination with interferons in the treatment of malignant pleural mesothelioma. Br J Cancer. 1999;80:1781-5.

20. Byrne MJ, Davidson JA, Musk AW, Dewar J, van Hazel G, Buck $\mathrm{M}$, et al. Cisplatin and gemcitabine treatment for malignant pleural mesothelioma: a phase II study. J Clin Oncol. 1999;17:25-30.

21. Nowak AK, Byrne MJ, Williamson R, Ryan G, Segal A, Fielding $\mathrm{D}$, et al. A multicenter phase II study of cisplatin and gemcitabine for malignant mesothelioma. Br J Cancer. 2002;87:491-6.

22. van Haarst JMW, Baas P, Manegold Ch, Schouwink JH, Burgers JA, de Bruin HG, et al. Multicenter phase II study of gemcitabine and cisplatin in malignant pleural mesothelioma. $\mathrm{Br} \mathrm{J}$ Cancer. 2002;86:342-5.

23. Scagliotti GV, Shin DM, Kindler HL, Vasconcelles MJ, Keppler U, Manegold C, et al. Phase II study of pemetrexed with and without folic acid and vitamin B12 as front-line therapy in malignant pleural mesothelioma. J Clin Oncol. 2003;21:1556-61.
24. Manegold C, Symanowski J, Gatzemeier U, Reck M, von Pwel J, Kortsik C, et al. Second-line (post-study) chemotherapy received by patients treated in the phase 3 trial of pemetrexed plus cisplatin versus cisplatin alone in malignant pleural mesothelioma. Ann Oncol. 2005;16:923-7.

25. Porta C, Zimatore M, Bonomi L, Imarisio I, Paglino C, SartoreBianchi A, et al. Raltitrexed-oxaliplatin combination chemotherapy is inactive as second-line treatment for malignant pleural mesothelioma patients. Lung Cancer. 2005:48:429-34.

26. van Klaveren RJ, Aerts JG, de Bruin H, Giaccone G, Manegold $C$, van Meerbeeck JP. Inadequacy of the RECIST criteria for response evaluation in patients with malignant pleural mesothelioma. Lung Cancer. 2004;43:63-9.

27. Monetti F, Casanova S, Grasso A, Cafferata MA, Ardizzoni A, Neumaier CE. Inadequacy of the new response evaluation criteria in solid tumors (RECIST) in patients with malignant plural mesothelioma: report of four cases. Lung Cancer. 2004;43:71-4.

28. Byrne MJ, Nowak AK. Modified RECIST criteria for assessment of response in malignant pleural mesothelioma. Ann Oncol. 2004;15:257-60.

29. Armato III SG, MacMahon H, Oxnard GR, Croteau CL, Vogelzang NJ. Radiologic assessment of mesothelioma. In: Pas HI, Vogelzang NJ, Carbone M, editors. Malignant Mesothelioma. New York: Springer; 2005. p. 433-53.

30. Ceresoli GL, Chiti A, Zucali PA, Rodari M, Lutman RF, Salamina S, et al. Early response evaluation in malignant pleural mesothelioma by positron emission tomography with $[18 \mathrm{~F}]$ fluorodeoxyglucose. J Clin Oncol. 2006;24:4587-93.

31. Carmichael J, Degraff WG, Gamson J, Russo D, Gazdar AF, Levitt ML, et al. Radiation sensitivity of human lung cancer cell lines. Eur J Cancer Clin Oncol. 1989;25:527-34.

32. Boutin C, Rey F, Viallat JR. Prevention of malignant seeding after invasive diagnostic procedures in patients with pleural mesothelioma. A randomized trial of local radiotherapy. Chest. 1995; 108:754-8.

33. do Graat-Strukowska, van der Zee, van Putten W, Senan S. Factors influencing the outcome of radiotherapy in malignant mesothelioma of the pleura-a single-institute experience with 189 patients. Int J Radit Oncol Biol Phys. 1999;43:511-6.

34. Bydder S, Phillips M, Joseph DJ, Cameron F, Spry NA, DeMelker Y, et al. A randomized trial of single-dose radiotherapy to prevent procedure tract metastasis by malignant mesothelioma. Br J Cancer. 2004;91:9-10.

35. Rusch VW, Rosenzweig K, Venkatraman E, Leon L, Raben A, Harrison L, et al. A phase II trial of surgical resection and adjuvant high-dose hemithoracic radiation for malignant pleural mesothelioma. J Thorac Cardiovasc Surg. 2001;122:788-95.

36. Zierhut D, Gutwein S, Munter MW, Woger H, Debus J. Radiation therapy of mesothelioma: the Heidelberg experience and future aspects. Lung Cancer. 2004;455:585-91.

37. Forster KM, Smythe WR, Starkschall G, Liao Z, Takanaka T, Kelly JF, et al. Intensity-modulated radiotherapy following extrapleural pneumonectomy for the treatment of malignant mesothelioma: clinical implementation. Int J Radiat Oncol Biol Phys. 2003;55:606-16.

38. Allen AM, Czerminska M, Jänne PA, Sugarbaker DJ, Bueno R, Harris JR, et al. Fatal pneumonitis associated with intensitymodulated radiation therapy for mesothelioma. Int J Radiat Oncol Biol Phys. 2006;65:640-5.

39. Jang SY, Liu HH, Wang X, Vassiliev ON, Siebers JV, Dong L, et al. Dosimetric verification for intensity-modulated radiotherapy of thoracic cancers using experimental and Monte Carlo approaches. Int J Radat Oncol Biol Phys. 2006;66:939-48.

40. Kukreja J, Jaklitsch MT, Wiener DC, Sugarbaker DJ, Burgers S, Baas P. Malignant pleural mesothelioma: overview of the North 
American and European experience. Thorac Surg Clin. 2004; $14: 435-45$.

41. Treasure T, Waller D, Swift S, Peto J. Radical surgery for mesothelioma. BMJ. 2004;328:237-8.

42. Sugarbaker DJ, Flores RM, Jaklitsch MT, Richards WG, Strauss GM,Corson JM, et al. Resection margins, extrapleural nodal status, and cell type determine postoperative long-term survival in trimodality therapy of malignant pleural mesothelioma: results in 183 patients. J Thorac Cardiovasc Sur. 1999;117:54-63; discussion $63-5$.
43. McCormack PM, Nagasaki F, Hilaris BS, Martini N. Surgical treatment of pleural mesothelioma. J Thorac Cardiovasc Sur. 1982;84:834-42.

44. Weder W, Kestenholz P, Tavema C, Bodis S, Lardinois D, Jerman M, et al. Neoadjuvant chemotherapy followed by extrapleural pneumonectomy in malignant pleural mesothelioma. J Clin Oncol. 2004;22:3451-7. 